\title{
LA ONTOLOGÍA DE LOS PROBLEMAS DE DIFERENCIA Y REPETICIÓN
}

\author{
Simón Díez Montoya* \\ doi: 10.11144/Javeriana.uph37-74.opdr
}

\section{RESUMEN}

Este artículo tiene como objetivo explicitar la ontología de los problemas que se propone en el magnum opus de Deleuze, Diferencia y repetición (1968). Por "ontología de los problemas" me refiero a la conceptualización de los problemas como primordialmente ontológicos, antes que epistemológicos. Este ser-problemático es el objeto de las Ideas, que son el núcleo duro de la ontología deleuziana expuesta en la actualización de lo virtual y sus cuatro aspectos (diferenciación [différentiation], individuación, dramatización, diferenciación [différenciation]). Por esta razón, una comprensión más profunda de la ontología de los problemas funciona como el preludio más adecuado para un abordaje cuidadoso de toda la ontología deleuziana. Además, el artículo resalta las fuentes filosóficas y científicas de las cuales bebe Deleuze para construir su ontología de los problemas. Especialmente notable es el uso de algunos metafísicos del cálculo diferencial (Bordas-Demoulin, Maïmon, Hoëne-Wronski) y algunos matemáticos prominentes (Carnot, Abel, Galois, Riemann, Lautman) del siglo XIX y temprano siglo XX.

Palabras clave: Deleuze; problemas; Ideas; ontología; Diferencia y repetición

Universidad del Rosario, Bogotá, Colombia.

Correo electrónico: simon.diez.mon@gmail.com

Para citar este artículo: Díez Montoya, S. (2020). La ontología de los problemas de Diferencia y repetición. Universitas Philosophica, 37(74), 77-100. ISSN 0120-5323, ISSN en línea 2346-2426. doi: 10.11144/Javeriana.uph37-74.opdr 


\title{
THE ONTOLOGY OF PROBLEMS IN DIFFERENCE AND REPETITION
}

\begin{abstract}
This paper outlines the ontology of problems in Deleuze's magnum opus, Difference and Repetition (1968). By ontology of problems I refer to the conceptualization of problems as a fundamentally ontological-not epistemological-subject. This problematic-being is the object of Ideas, which are at the core of Deleuzian ontology, as shown in the actualization of the virtual and its four aspects: differentiation [différentiation], individuation, dramatization, and differentiation [différenciation]. For this reason, a deeper comprehension of the ontology of problems serves as the most adequate prelude for a sound approach to all of Deleuzian ontology. In addition, this article highlights the philosophical and scientific sources upon which Deleuze draws in order to build his ontology of problems. Of special note are his references to several metaphysicians of differential calculus (Bordas-Demoulin, Maïmon, Hoëne-Wronski) and prominent mathematicians (Carnot, Abel, Galois, Riemann, Lautman) of the $19^{\text {th }}$ and $20^{\text {th }}$ centuries.

Keywords: Deleuze; problems; Ideas; ontology; Difference and Repetition
\end{abstract}




\section{Introducción}

Las preguntas... se fabrican. Y si no os dejan fabricar vuestras preguntas, con elementos tomados de aquí y de allí, si os las "plantean", poco tenéis que decir. El arte de construir un problema es muy importante: antes de encontrar una solución, se inventa un problema, una posición de problema (Deleuze \& Parnet, 1977/1980, p. 5).

Esto afirma el filósofo Gilles Deleuze en una entrevista hecha por solicitud de la periodista Claire Parnet. De entrada, parece una curiosa manera de iniciar una entrevista ("nada de andar 'planteando' preguntas"); sin embargo, de fondo, muestra una consigna que Deleuze mantendrá a lo largo de su obra filosófica: los problemas no preexisten a su planteamiento; lo primero que hace un filósofo es plantear sus propios problemas ${ }^{1}$.

La cuestión del "problema" en Diferencia y repetición no comienza con una definición, sino con dos figuras: la del alumno y la del autodidacta (Deleuze, 1968/2002, p. 290-292). Al alumno un maestro le plantea un problema (por ejemplo, $2 \times 2=\dot{e}$ ?), el cual ya ha sido calcado previamente sobre una respuesta (por ejemplo, $2 \times 2=4$ ). $\mathrm{Al}$ autodidacta, una situación lo fuerza a pensar y a plantear un problema (por ejemplo, ¿cómo me muevo y compongo mi cuerpo para pasearme a la par que la ola?). El primero vuelve el problema una insuficiencia epistemológica (subjetiva) que debe ser suplida, de ahí las representaciones comunes de los problemas como vacíos que se llenan, como obstáculos que se superan, o como dudas que se resuelven (a saber, si el alumno acierta, el problema desaparece; si yerra, el maestro le señala su error). El segundo vuelve el problema una positividad ontológica (objetiva) que debe determinarse, de ahí su permanencia como una posición de problema o unas condiciones problemáticas capaces de

1 Antes de Diferencia y repetición (2002), Deleuze explora la noción de problema de la mano del método de la intuición de Henri Bergson en El bergsonismo (1966/2017). Tal método consta de tres reglas: la primera es aplicar la prueba de lo verdadero y lo falso a los problemas mismos; la segunda es denunciar las ilusiones y descubrir las verdaderas diferencias de naturaleza; y la tercera es plantear y resolver problemas en función del tiempo, más que del espacio. Llama la atención este descubrimiento conceptual con el cual puede comenzar a extirpar toda negatividad de la manera como se piensan los problemas, pues, en vez de ser un momento secundario en la actividad filosófica, el planteamiento de los problemas es el acto inaugural de toda filosofía. 
engendrar casos de solución diferenciados (a saber, el autodidacta puede producir soluciones divergentes o intentos para el mismo problema, y las soluciones se adjuntan unas a otras: por ejemplo, mover mis brazos y mis piernas de tal o cual manera, usar una tabla de surf, dejarme alcanzar por la ola y posicionarme para que ella me lleve, etc.; de manera que el problema nunca desaparece más bien se determina progresivamente con cada solución).

¿Cómo superar el lastre negativo y subjetivo que tiene la noción de problema y volverlo más bien positivo y objetivo? Tal primacía ontológica de los problemas puede designarse, justamente, como una "ontología de los problemas": una conceptualización sobre el Ser mismo como problemático. Entonces, para dar respuesta a qué es un problema, debemos ir al corazón mismo de la ontología deleuziana: su concepción de la Idea como estructura genética que se encarna en la producción de las cosas mismas, producción que se nombra como la "actualización de lo virtual” y que consta de cuatro aspectos: diferenciación [différentiation], individuación, dramatización, diferenciación [différenciation] $]^{2}$. Para adentrarse en los problemas, Deleuze pasa por una serie de fuentes inusuales para la historia de la filosofía: los metafísicos del cálculo diferencial en el siglo XVIII y XIX y los matemáticos de la teoría de los problemas del siglo XIX y temprano siglo XX.

Con esto en mente, este artículo propone seguir la pista de los problemas en Diferencia y repetición como una clave de lectura que abre las puertas a una comprensión más completa de la ontología deleuziana ${ }^{3}$. Primero, veremos, con

2 En la versión original francesa de Diferencia y repetición, Deleuze distingue entre différentiation ( $\operatorname{con} t)$ como la determinación virtual de la Idea en tanto sistema de relaciones diferenciales y singularidades y la différenciation ( $\operatorname{con} c$ ) como la encarnación de la Idea en los términos y las especies que actualizan las relaciones diferenciales y en las formas y partes que actualizan las singularidades.

3 En la literatura secundaria más reciente, el concepto de "problema" ha sido abordado mucho más a partir de las herencias filosóficas y científicas de Deleuze que a partir de su funcionamiento dentro de la ontología deleuziana. Por ejemplo, James Bahoh (2019) rastrea las influencias heideggerianas en Diferencia y repetición a través de la incorporación de Albert Lautman (quien fuera lector de Martin Heidegger); Giuseppe Bianco (2018) explora una historia del problema muy anterior a las formulaciones de Deleuze: desde sus orígenes en la geometría griega, pasando por su renacimiento moderno en Kant y su recepción en la academia alemana y francesa, llegando hasta Deleuze; Simon Duffy (2009) explora la "metafísica de la lógica" del filósofo de las matemáticas Albert Lautman como la base de la noción de Idea en Deleuze; Ellie During (2004), a partir de la noción de problema, traza el linaje de la tradición epistemológica francesa antipositivista inaugurada por Bergson en pensadores como Bachelard, Canguilhem, Merleau-Ponty, Foucault y Deleuze; Félix Ernesto 
Immanuel Kant (1724-1804), por qué las Ideas son problemas, o mejor, por qué los problemas son "el" objeto de las Ideas. Segundo, de la mano de Jean BordasDemoulin (1798-1859), Salomon Maïmon (1753-1800) y Józef Maria HoëneWronski (1776-1853), abordaremos la transformación de la Idea kantiana en una diferencial que excede el sentido meramente matemático de esta y arriba a una verdadera metafísica. Tercero, con ayuda de Nicolas Léonard Sadi Carnot (1796-1832), Neils Henrik Abel (1802-1829), Évariste Galois (1811-1832) y Albert Lautman (1908-1944), aprovecharemos la teoría matemática de los problemas como un genuino recurso ontológico: hay una diferencia de naturaleza entre el problema y sus soluciones; igualmente, el problema es al mismo tiempo trascendente (en tanto no desaparece) e inmanente (en tanto se determina) con respecto a sus soluciones. Cuarto, a partir de Bernhard Riemman (1826-1866), veremos que la caracterización de la Idea como variedad o multiplicidad permite su concepción como estructura genética universal, lo cual abre la puerta a una dialéctica propiamente deleuziana. Finalmente, vemos la síntesis deleuziana de todas estas líneas en la manera como se describe la actualización de lo virtual: se trata, a la vez, de la encarnación de la Idea y de la solución progresiva y divergente de un problema.

\section{Kant: las Ideas son problemáticas y los problemas son Ideas}

Si LA RAZÓn ENGENDRA SUS PROPIAS ILUSIONES (como cree Kant), entonces estamos ante una facultad de plantear problemas (como cree Deleuze): más allá de la posibilidad de dar con falsos problemas ${ }^{4}$, debemos ver la capacidad misma de

González Geraldino (2018) utiliza el concepto de problema para enriquecer el análisis funcional, creando una especie de teoría de sistemas luhmanno-deleuziana en tanto cada subsistema funcional (Luhmann) estaría anclado a un problema (Deleuze); por último, Andrew Wasser (2017) toma la impronta de la conferencia de Deleuze ¿Cómo reconocer el estructuralismo? y se pregunta “¿cómo reconocer un problema?", llegando de manera análoga a seis aspectos del problema: (1) los problemas son afirmativos; (2) los problemas irrumpen el sentido común; (3) la condición del problema es la inmanencia; (4) los problemas se determinan intrínsecamente; (5) los problemas falsos expresan el carácter del pensamiento; y (6) los problemas son el objeto de la crítica.

4 Los falsos problemas, muestra Deleuze (2017) siguiendo a Bergson, vienen de dos modos: o bien son inexistentes, porque confunden el "menos" con el "más" (e.g. ser y no-ser se presentan como equivalentes, aun cuando este último tiene de más una operación lógica de negación y un motivo 
plantearlos. Asimismo, "toda solución supone un problema, es decir, la constitución de un campo sistemático unitario que orienta y subsume las investigaciones o las interrogaciones, de tal modo que las respuestas, a su vez, forman precisamente casos de solución" (Deleuze, 2002, p. 258). Debemos llegar a ver los verdaderos problemas como Ideas, pues estos no solo no desaparecen con sus soluciones, sino que, sin problemas, las soluciones no podrían llegar siquiera a ser.

Por su parte, así como los problemas tienen un valor objetivo, también las Ideas tienen un objeto. Deleuze retoma de Kant una caracterización tripartita de las Ideas problemáticas para mostrar, en últimas, que los problemas en sí mismos son los objetos de las Ideas. En primer lugar, las Ideas son al mismo tiempo objetivas e indeterminadas. Lejos de reducir los problemas a una insuficiencia epistemológica subjetiva, Kant los dota de una estructura positiva que opera como un foco u horizonte. En segundo lugar, las Ideas se vuelven indirectamente determinadas cuando el problema recubre los objetos de la experiencia en una unidad sistemática, a la vez que estos lo determinan a partir de las relaciones que se establecen a su alrededor. En tercer lugar, el objeto de la Idea -el problema- carga consigo un ideal de determinación completa e infinita; pues simultáneamente especifica y amplía los conceptos del entendimiento. Para resumir, gracias a los problemas como objetos indeterminados (primer momento de la Idea: indeterminación) es que podemos luego conferirles una unidad sistemática a los objetos de la experiencia (segundo momento de la Idea: determinación indirecta) y una especificación infinita a los conceptos del entendimiento (tercer momento de la Idea: determinación infinita).

No obstante, Deleuze insiste en que Kant no fue lo suficientemente lejos en su exposición de las Ideas problemáticas. Kant sigue manteniendo a dos de los tres momentos como "externos" a las Ideas mismas: si la determinación indirecta solo es posible en relación con los objetos de la experiencia y la determinación completa con los conceptos del entendimiento, entonces las Ideas no pueden determinarse por sí solas. Según Deleuze, esto hace que Kant llegue apenas al punto de vista del "condicionamiento", como le reprochaban los neokantianos,

psicológico particular); o bien están mal planteados, porque se formulan a partir de un mal análisis de los mixtos (es decir, confunden diferencias de grado con diferencias de naturaleza, como intentar cuantificar la sensación). 
ignorando el punto de vista de la "génesis". Hemos de ver cómo no hay "en la Idea ninguna identificación ni confunsión, sino una unidad objetiva problemática interna, de lo indeterminado, de lo determinable y de la determinación" (Deleuze, 2002, p. 260).

\section{Bordas-Demoulin, Maïmon y Hoëne-Wronski: una metafísica del cálculo diferencial}

Para Deleuze (2002), "en las antiguas interpretaciones del cálculo diferencial, tildadas de bárbaras o precientíficas, hay un tesoro que debe ser desprendido de su ganga infinitesimal” (p. 260). ¿Quiénes, en la historia esotérica de la filosofía diferencial, como la llama Deleuze, se han tomado en serio el símbolo $d x$ (la diferencial)? La respuesta conduce a tres extraños poskantianos que reinterpretan respectivamente, según la lectura deleuziana, los tres momentos de la Idea en Kant: primero, Bordas-Demoulin, a través de una lectura platónica del cálculo, muestra cómo los símbolos $d x y d y$ transforman el momento de indeterminación en un principio de determinabilidad; segundo, Maïmon, a través de una lectura leibniziana del cálculo, muestra cómo la relación $d y / d x$ transforma el momento de determinación indirecta en un principio de determinación recíproca; $\mathrm{y}$ tercero, Hoëne-Wronski, a través de una lectura kantiana y mística del cálculo, muestra cómo los valores de $d y / d x$ transforman el momento de determinación infinita en un principio de determinación completa. Ante esta breve historia, la tarea de Deleuze es mostrar a la diferencial $(d x)$ como Idea y a la Idea como problema.

Para empezar, los símbolos $d x$ y $d y$ no expresan ni una cantidad fija de la intuición, ni una cantidad variable del entendimiento, sino la cuantitabilidad misma. Es decir, están completamente indeterminados: $d x$ no es nada en relación con $x$, así como $d y$ no es nada en relación con $y$. La interpretación filosófica de Bordas-Demoulin es que $d x$ y $d y$ no son ni particulares (cantidades fijas de la intuición) ni generales (cantidades variables del entendimiento), sino puramente universales (matemáticamente, $0 / 0$ no es igual a 0 , sino que muestra una relación pura e irreducible a una cantidad fija o variable). En palabras de Deleuze, aunque $d x$ y $d y$ están completamente indiferenciados en lo particular y lo general, están completamente diferenciados [différentiés] en lo universal. Por eso las Ideas no son ni particulares ni generales, sino universales. 
En seguida, la relación $d y / d x$ expresa que, si bien $d x$ y $d y$ están indeterminados en relación con $x$ o $y$, en relación entre sí son determinables: $d y / d x$ no es una fracción cualquiera entre cantidades fijas o variables, sino la pura relación de reciprocidad en tanto cada término existe solo en su relación con el otro. Concretamente, esta determinabilidad recíproca implica una determinabilidad de la relación misma: una síntesis recíproca. Para Deleuze, la pregunta es “¿bajo qué forma la relación diferencial es determinable?” (2002, p. 263). La respuesta complicada es que $d y / d x$ no es una simple función primitiva del cálculo, sino el elemento puro de cualitabilidad.

Es en ese sentido que la Idea tiene por objeto la relación diferencial: integra entonces la variación, pero de ningún modo como determinación variabale de una relación que se supone constante ("variabilidad"), sino, por el contrario, como grado de variación de la relación misma ("variedad”), al que corresponde (Deleuze, 2002, p. 264).

En una vena más filosófica, habría que decir que la Idea no es un concepto que varía, sino una variedad misma que integra la dependencia recíproca de los grados de la relación diferencial y también la dependencia recíproca de las relaciones mismas. De acuerdo con Maïmon, ir más lejos que Kant en este punto implica concebir "la síntesis recíproca de las relaciones diferenciales, como fuente de la producción de los objetos reales" (Maïmon citado indirectamente en Deleuze, 2002, p. 265). Esta interpretación claramente traspasa el límite epistemológico o regulativo conferido por Kant a las Ideas y las lleva a un terreno ontológico y genético. Esta última acepción ve a las Ideas como sistemas de relaciones diferenciales entre elementos genéticos determinados recíprocamente.

Finalmente, los valores de $d y / d x$ expresan la pura potencialidad de la relación diferencial. En este punto, Hoëne-Wronski se opone directamente a las interpretaciones puramente matemáticas del cálculo que encuentra en Joseph-Louis Lagrange (1736-1813) (series de Taylor) y Carnot (compensación de errores), pues considera que presuponen ya una metafísica del cálculo no especificada (HoëneWrosnki citado indirectamente en Deleuze, 2002). De hacerlo, se darían cuenta de que "las diferenciales no corresponden ciertamente a ninguna cantidad generada, sino que son una regla incondicionada para la génesis del conocimiento de la cantidad, y para la generación de las discontinuidades que constituyen su 
materia o para la construcción de series" (Deleuze, 2002, p. 267). Los valores diferenciales son por eso potencialidad, es decir, la relación diferencial es una "diferencia ideal" (Idea). Si el principio de determinación recíproca de la diferencial $d y / d x$ compromete las relaciones genéticas capaces de engendrar objetos, entonces el principio de determinación completa de los valores de la diferencial $d y / d x$ compromete la distribución de los puntos singulares que componen una forma específica de los objetos.

Para resumir, la Idea diferencial transforma los tres momentos de la Idea kantiana en tres principios para su determinación interna, problemática y objetiva: primero, un principio de determinabilidad (que expresa su pura cuantitabilidad) de manera que la Idea no es ni general ni particular, sino universal; segundo, un principio de determinación recíproca (que expresa su pura cualitabilidad), de manera que la Idea integra la pura relación diferencial; tercero, un principio de determinación completa (que expresa su pura potencialidad), de manera que la Idea tiene tal o cual distribución de puntos singulares o singularidades con un verdadero potencial genético. Con esto, vemos ya esbozada la síntesis deleuziana de esta extraña historia de la filosofía "diferencial": la diferencial en tanto Idea que expresa una pura cuantitabilidad, cualitabilidad y potencialidad. Bajo esta luz, ahora intensificada por la metafísica del cálculo neokantiana, las Ideas aparecen como universales concretos, compuestos a su vez por relaciones diferenciales y por distribuciones de puntos singulares.

\section{Carnot, Lautman, Abel y Galois: una dialéctica de los problemas}

LA METAFísica del CÁlCUlo, reconstruida a partir de Bordas-Demoulin, Maïmon y Hoëne-Wronski, no solo postula una concepción verdaderamente ontológica de las Ideas como universales, genéticas y reales, sino que también deja una pregunta abierta: "¿por qué, técnicamente, las diferenciales son despreciables y deben desaparecer del resultado?” (Deleuze, 2002, p. 269). Desde una interpretación finitista del cálculo, para Carnot es claro: las diferenciales no son sino "auxiliares" que muestran las condiciones de un problema al cual responde una ecuación dada que, en su solución, puede ignorar estas auxiliares (Carnot citado indirectamente en Deleuze 2002). No obstante, Deleuze recurre a Carnot, a pesar de su finitismo, por su rica noción de problema o de condiciones 
de un problema: lejos de nombrar un error útil, estas auxiliares muestran la diferencia de naturaleza entre el problema y sus soluciones. A la vez trascendente e inmanente con respecto a sus soluciones, un problema, para Carnot, plantea las condiciones objetivas bajo las cuales pueden engendrarse sus soluciones, al mismo tiempo que continúa diferenciándose a través de ellas.

Esta dialéctica de los problemas, como la llama Deleuze, nombra una sola cosa: antes que matemáticos, físicos, químicos, biológicos o sociales, los problemas son dialécticos, pues expresan la objetividad del elemento problemático como tal. Análoga a la de la Idea, esta objetividad tiene una caracterización tripartita a partir de la filosofía de las matemáticas de Lautman:

Conforme a las tesis generales de Lautman, el problema tiene tres aspectos: su diferencia de naturaleza con las soluciones; su trascendencia en relación con las soluciones que genera a partir de sus propias condiciones determinantes; su inmanencia a las soluciones que lo recubren, estando el problema mejor resuelto cuanto más se determina (Lautman citado indirectamente en Deleuze, 2002, pp. 178-179).

Para estar completa, la dialéctica de los problemas no solo afirma la objetividad del elemento problemático y sus diferencias de naturaleza, trascendencia e inmanencia con respecto a las soluciones, sino que también debe revertir la manera habitual de concebir la relación entre el problema y sus soluciones. Un problema no es verdadero porque sea soluble, antes bien, es soluble porque es verdadero 5 : la solubilidad se deriva de las condiciones de un problema. Deleuze (2002) le atribuye esta "revolución copernicana" en la teoría matemática de los problemas a Abel: "es preciso determinar condiciones de problemas que especifiquen progresivamente campos de resolubilidad, de tal modo que 'el enunciado contenga el germen de la solución"” (pp. 273-274). El trabajo de Galois sigue la misma línea: "a partir de un cuerpo de base $(\mathrm{R})$, las adjunciones sucesivas a ese cuerpo (R', R’, R’”) permiten una distinción cada vez más precisa de las raíces de una ecuación, por limitación progresiva de las susticiones posibles. Hay,

5 En esto Deleuze (2017) continúa siendo profundamente bergsoniano: no solo se crean los problemas, también se crean (y no se descubren) las soluciones, precisamente como una consecuencia de este primer acto creativo. 
por consiguiente, una cascada de "resolventes parciales" o un encabalgamiento de "grupos" que hacen derivar las soluciones de las condiciones del problema" (Deleuze, 2002, p. 274). En resumen, los verdaderos problemas dejan atrás su carácter de insuficiencia epistemológica - vacío, no-conocimiento, duda- para volverse una positividad ontológica caracterizada en virtud de un discernimiento progresivo a través de sucesivas soluciones.

La última síntesis de la dialéctica de los problemas vuelve sobre el paralelo Ideas-problemas; en este punto vale la pena citar extensamente al propio Deleuze (2002):

La Idea dialéctica, problemática, es un sistema de relaciones diferenciales entre elementos genéticos. Hay diferentes órdenes de Ideas, supuestos los unos por los otros, según la naturaleza ideal de las relaciones y los elementos considerados (Idea de Idea, etc.). Esas definiciones no tienen todavía nada de matemático. Las matemáticas surgen con los campos de solución en los cuales se encarnan las Ideas dialécticas de último orden, y con la expresión de los problemas relativa a esos campos. Otros órdenes en la Idea se encarnan en otros campos y en otras expresiones que corresponden a otras ciencias. Es así como, a partir de los problemas dialécticos y de sus órdenes, se produce una génesis de dominios científicos diversos. El cálculo diferencial, en el sentido más preciso, no es sino un instrumento matemático que, aun en su dominio, no representa necesariamente la forma más acabada de la expresión de los problemas y de la constitución de las soluciones en relación con el orden de las Ideas dialécticas que encarna. No por eso tiene un sentido menos amplio, por el cual debe designar universalmente el conjunto del compuesto Problema o Idea dialéctica - Expresión científica de un problema - Instauración del campo de solución. Más generalmente debemos concluir que no hay dificultad relativa a una pretendida aplicación de las matemáticas, y sobre todo del cálculo diferencial o de la teoría de grupos, a otros dominios. Más bien cada dominio generado, y en el que se encarnan las Ideas dialécticas de tal o cual orden, posee su propio cálculo. Las Ideas siempre tienen un elemento de cuantitabilidad, de cualitabilidad, de potencialidad; procesos de determinabilidad, de determinación recíproca y de determinación completa; distribuciones de puntos notables y ordinarios, y cuerpos adjuntos que forma la progresión sintética de una razón suficiente (pp. 275-276). 
En la cita, vemos la insistencia de Deleuze por separar la dialéctica de los problemas del cálculo diferencial como dominio matemático específico. Si esta dialéctica ha de tener un carácter realmente universal, entonces no puede ser meramente matemática. Más aún, esta dialéctica implica una "génesis de diversos dominios científicos", pues una Idea dialéctica (o, lo que es igual, un Problema) es un universal concreto compuesto de "relaciones diferenciales entre elementos genéticos" capaces de engendrar distribuciones de "puntos distintivos y ordinarios" y "campos adjuntos de progresión", todo lo cual exige especificarse en órdenes (como las matemáticas) y dominios (como el cálculo diferencial). Justamente la "aventura de las Ideas", como explica Deleuze (2002), es buscar, para cada Idea, sus órdenes, dominios y campos relativos de encarnación, es decir, su propio cálculo (ya no necesariamente matemático, sino una suerte de "álgebra del pensamiento puro") (p. 276).

\section{Riemann: la Idea dialéctica o problemática como multiplicidad}

Además de tomar elementos de la teoría matemática de los problemas, Deleuze recurre a Riemann y su noción de variedad o multiplicidad para alejarse de toda concepción esencialista de la Idea como 'lo que hace que la cosa sea lo que es. Una Idea es una multiplicidad: ni uno, ni múltiple, sino una multiplicidad. Esta insistencia en olvidarse de una multiplicación de lo Uno y al mismo tiempo de una unificación de lo Múltiple se vuelve un refrán deleuziano: "la multiplicidad no debe designar una combinación de lo múltiple y lo uno, sino, por el contrario, una organización propia de lo múltiple como tal, que de ningún modo tiene necesidad de unidad para formar un sistema" (Deleuze, 2002, pp. 276-277). En su adaptación filosófica, la multiplicidad puede entenderse como la base sustantiva de la ontología deleuziana. En efecto, es un cambio de pregunta: ya no se trata de inquirir por el qué o la esencia de las cosas, sino por el en qué casos o el estudio singular de la manera de ser de cada cosa. Al fin y al cabo, cualquier cosa "es una multiplicidad en tanto encarna la Idea" (Deleuze, 2002, p. 277).

A la luz de lo anterior, ¿qué significa pensar? Pues, pensar es "el arte de las multiplicidades, el arte de captar en las cosas las Ideas, los problemas que encarnan, y de captar las cosas como encarnaciones, como casos de solución para problemas de Ideas" (Deleuze, 2002, p. 277). En este punto, se hace evidente la 
metamorfosis ontológica de la noción de problema: el Ser es un problema y los seres son sus casos de solución progresivamente diferenciados.

Hablamos de una multiplicidad cuando se cumplen tres condiciones (Deleuze, 2002). Primero, una multiplicidad está compuesta por elementos indeterminados y virtuales que constituyen un potencial. Segundo, estos elementos pueden determinarse recíprocamente a partir de la progresiva yuxtaposición de regiones vecinas que expresan una relación ideal; en otras palabras, una multiplicidad se define intrínsecamente a través de las conexiones entre cada elemento y no extrínsecamente por su inserción en un plano superior. Tercero, esta relación ideal se actualiza en diversas relaciones espaciotemporales concretas a la vez que estas conexiones se encarnan en varios términos y formas puntuales. Comprendida de esta manera, la multiplicidad permite conciliar la estructura con su génesis; pues una Idea es una estructura virtual que se encarna en relaciones y términos actuales. En palabras de Deleuze (2002):

Basta comprender que la génesis no va de un término actual, por más pequeño que sea, a otro término actual en el tiempo; sino de lo virtual a su actualización, es decir, de la estructura a su encarnación, de las condiciones de los problemas a los casos de solución, de los elementos diferenciales y de sus relaciones ideales a los términos actuales y a las relaciones reales diversas que constituyen en cada momento la actualidad del tiempo (pp. 278-279).

Así pues, queda expuesta la arquitectura conceptual deleuziana y todos sus paralelismos formales: la Idea-Problema-Multiplicidad se actualiza-resuelveencarna en términos-casos de solución-cosas. Esta es la ontología de toda Idea. No importa si se trata de una Idea matemática, física, química, biológica o social. Para Deleuze, lo fundamental no es saber qué son las Ideas (eso lo podemos definir de antemano: son problemas, multiplicidades, estructuras, virtualidades, etc.); sino estudiar, para cada caso, su actualización concreta.

De cierta manera, incluso, todas las Ideas coexisten; pues no solo hay una variedad de Ideas, también cada Idea es una variedad con tres dimensiones o subvariedades (Deleuze, 2002). En primer lugar, hay variedades ordinales de acuerdo con la naturaleza de los elementos y sus relaciones: órdenes matemáticos, físicos, químicos, biológicos, sociales, etc. Sin embargo, no se trata de concebirlos como interioridades cerradas; más bien estos órdenes pueden disolverse 
"hacia arriba" o reflejarse "hacia abajo" entre sí. En segundo lugar, hay variedades caracteristicas correspondientes con la distribución de los puntos singulares de cada orden: para cada uno, debe mostrarse cómo la diversidad de puntos ordinarios se reúne en torno a puntos singulares marcados (por ejemplo, la variedad de animales ordenados desde el punto de vista de la composición [Deleuze, 2002]). Finalmente, hay variedades axiomáticas que corresponden a axiomas comunes entre órdenes diferenciados (por ejemplo, la adición de números reales y la composición de desplazamientos [Deleuze, 2002]). Deleuze (2002) llama este estado de coexistencia de las Ideas, en virtud del dinamismo entre las subvariedades, perplicación. No se trata de marcar una perplejidad o incompletitud de las Ideas, sino de mostrar la imbricación Ideas-problemas: "la manera en que los problemas están objetivamente determinados por sus condiciones a participar los unos en los otros, de acuerdo con las exigencias circunstanciales de la síntesis de las Ideas" (Deleuze, 2002, p. 284).

Por eso, las Ideas no son esencias: no preguntan por el qué, sino por el cómo, dónde, cuándo, quién, cuánto, en qué casos. Asimismo, "[el] problema pertenece al orden del acontecimiento. No solo porque los casos de solución surjan como acontecimientos reales, sino porque las mismas condiciones del problema implican acontecimientos, secciones, ablaciones, adjunciones" (Deleuze, 2002, p. 286). En efecto, pensar dialécticamente es afirmar que solo sabemos qué puede una Idea cuando la actualizamos en tales o cuales circunstancias, que solo entendemos qué puede un problema cuando abrimos su región problemática. Más aún, los problemas abren una doble serie eventual. Por un lado, sus condiciones problemáticas son eventos ideales a la vez trascendentes (porque no desaparecen) e inmanentes (porque continúan determinándose progresivamente) con respecto a los casos de solución. Por otro lado, dichos casos de solución emergen como eventos, son soluciones engendradas por las condiciones problemáticas.

En este sentido, los problemas atañen directamente al pensamiento (justamente eso significa "tener Ideas"). Como tal, el problema del pensamiento

[...] no está relacionado con el de esencia, sino con la evaluación de lo que tiene importancia y de lo que no la tiene, con la distribución de lo singular y de lo regular, de lo notable y lo ordinario: que se cumple por entero en lo inesencial o en la descripción de una multiplicidad, en relación con los 
eventos ideales que constituyen las condiciones de un "problema" (Deleuze, 2002, p. 287).

En particular, "la descripción de una multiplicidad" concierne el acto de vicedicción, a su vez entendido en dos procedimientos ligados a la caracterización de las condiciones de un problema: por una parte, la especificación de campos adjuntos, y, por otra, la condensación de singularidades. La primera indica que un problema no está bien planteando hasta que no se haya mapeado el campo del problema, ¿con qué órdenes y dominios debe estar "perplicado" para que sea soluble? La segunda indica que la distribución de puntos singulares o singularidades en las condiciones de un problema debe ser tal que fuerzan -literalmente- una solución, a saber, esta última debe aparecer como un verdadero evento. "Tener una Idea", entonces, designa una objetividad, una eventualidad real: "las Ideas, al igual que los Problemas, no solo están en nuestra cabeza, sino aquí y allá, en la producción de un mundo histórico actual" (Deleuze, 2002, p. 288).

\section{La realidad de las Ideas: la actualización de lo virtual}

LAS IDEAS no solo conciernen al movimiento del pensamiento en nuestras cabezas, sino también a la producción de las cosas mismas. Si hay una proposición ontológica transversal a toda la ontología deleuziana, es esta. Una comprensión profunda de lo anterior debe explorar minuciosamente todas las distinciones que hacen posible esta Idea (aquella según la cual lo virtual se actualiza):

Siempre es en relación con un problema diferenciado [différentié], en condiciones de problemas diferenciados [différentiées], que se opera una diferenciación [différenciation] en especies y partes, como correspondiendo a los casos de solución del problema. Siempre es un campo problemático el que condiciona una diferenciación [différenciation] en el medio donde se encarna (Deleuze, 2002, pp. 311-312).

Las Ideas se actualizan, los problemas se resuelven, lo diferentiado se diferencia en especies y partes: no importa cómo se designe, en cada caso, un potencial genético virtual es encarnado en términos y formas actuales.

A veces, sin embargo, la jerga filosófica confunde lo virtual y lo posible. Como lo posible se opone a lo real, asumimos que lo virtual también lo hace. 
Pero lo posible calca lo real para sustraerle toda la realidad; por eso, su única diferencia con respecto a lo real es que no se ha "realizado". Por el contrario, lo virtual no se opone a lo real, más bien tiene una manera de existencia propia. Lo virtual no se realiza, pues no es una instrucción o un plan prehecho, sino un problema; en vez, se actualiza. En efecto, para Deleuze (2002), siguiendo a Bergson, lo virtual es tan real como lo actual: "[lo] virtual [...] es el carácter de la Idea; es a partir de esta realidad que se produce la existencia, y se produce conforme con un tiempo y un espacio inmanentes a la Idea" (pp. 318-319). Se trata de la realidad de la estructura y "los elementos y relaciones diferenciales, y en los puntos singulares que le corresponden" (pp. 314-315). Por su parte, la realidad de lo actual es aquella de la génesis integral de las especies y las partes. Lo virtual se diferencia [différentie] en dos aspectos: las relaciones diferenciales y los puntos singulares. Asimismo, lo actual se diferencia [différencie] en dos aspectos: las especies diversas que actualizan las relaciones diferenciales y las partes distintas que actualizan los puntos singulares. Esto se puede ver claramente en el caso de organismos, para los cuales su misma vida se muestra como el planteamiento y la resolución de problemas locales:

La naturaleza de lo virtual es tal que actualizarse es diferenciarse para él. Cada diferenciación [différenciation] es una integración local, una solución local, que se compone con otras en el conjunto de la solución o de la integración global. Es así como, en lo viviente, el proceso de actualización se presenta a la vez como diferenciación local de las partes, formación global de un medio interior, solución de un problema planteado en el campo de constitución de un organismo. El organismo no sería nada si no fuera la solución de un problema, y lo mismo vale para cada uno de sus órganos diferenciados, así el ojo "resuelve" un problema de luz; pero nada en él, ningún órgano, se diferenciaría sin el medio interior dotado de una eficacia general o de un poder integrante de regulación (pp. 317-318).

En la actualización de lo virtual, la encarnación de una Idea, o la resolución de un problema, no tiene por qué aparecer nunca una identidad entre un elemento y otro: las especies y las partes actualizadas, las cosas encarnadas y los casos de solución, no se "asemejan" a sus virtualidades, Ideas o problemas. Por esto mismo, la génesis de estas estructuras constituye una verdadera producción: una 
misma virtualidad, una misma Idea, o un mismo problema, pueden engendrar series divergentes de actualizaciones, encarnaciones y soluciones.

Pero la dialéctica de los problemas llega aún más lejos. No solamente sabemos qué se actualiza, esto es, lo virtual, sino que debemos mostrar de igual forma cómo, cuándo, dónde, quién, cuánto y en qué casos se actualiza. Estas circunstancias de actualización se convierten, para Deleuze (2002), en pequeñas "agencias diferenciantes" que están detrás de las especies y partes actualizadas: se trata de los dinamismos espaciotemporales. Por ejemplo, la embriología contemporánea muestra que "la división de un huevo en partes es secundaria en relación con movimientos morfogenéticos de muy otra significación, aumento de superficies libres, estiramiento de capas celulares, invaginación por plegamiento, desplazamientos regionales de los grupos" (Deleuze, 2002, p. 322). Además de los dinamismos espaciotemporales, debemos considerar un sujeto larvario que los experimenta: "hay 'cosas' que solo el embrión puede hacer, movimientos que solo él puede emprender, o más bien soportar" (Deleuze, 2002, p. 323). Tanto el sujeto larval como sus dinamismos espaciotemporales indican la necesidad de un espacio y un tiempo propios de la diferenciación: una especie solo llega a serlo cuando se ha especificado, una parte solo llega a serlo cuando se ha partido. Las especies y partes ya actualizadas son el producto de lo que Deleuze llama una dramatización de la Idea, esto es, la producción de diferencias en extensión por medio de diferencias de intensidad. Más aún, estos espacios y tiempos intensivos solo pueden ser habitados por sujetos larvarios, sin los cuales nunca podrían diferenciarse espacios y tiempos extensivos habitados por sujetos gestados. No hay nada "que no pierda su identidad tal como es en el concepto, y su similitud tal como es en la representación, cuando se descubre el espacio y el tiempo dinámicos de su constitución actual" (Deleuze, 2002, p. 329). Entre lo virtual y su actualización está, pues, la dramatización.

Aun así, Deleuze (2002) se pregunta: “¿de dónde proviene este poder de la dramatización? ¿No es, bajo las especies y las partes, las cualidades y los números, el acto más intenso o más individual?” (p. 332). La respuesta conduce al último aspecto por considerar de la actualización de lo virtual: la individuación ("el acto más intenso o más individual”. ¿Cómo se ve esto? "Todo lo que pasa y aparece es correlativo de órdenes de diferencias, diferencias de nivel, de temperatura, de presión, de tensión, de potencial, diferencia de intensidad"(Deleuze, 2002, p. 333). 
¿Pero por qué, de entrada, no es evidente? La intensidad es inseparable de la extensión en la cual se desarrolla; por eso, de cierta forma, para descubrir lo verdaderamente intenso, debemos subir el río de la actualización a contracorriente, yendo de lo actual a su virtualización. Considerada en la extensión, la diferencia de intensidad siempre aparece ya diferenciada. Esto lo sabían ya los pioneros de la termodinámica, Carnot, Pierre Curie (1859-1906), Henri Louis Le Châtelier (1850-1936), a quienes Deleuze lee profusamente para mostrar que "la diferencia solo es razón suficiente de cambio en la medida en que ese cambio tiende a negarla" (p. 335). Esta negación de la diferencia se replica en el pensamiento cuando es invadido por un buen sentido "termodinámico": esa tendencia a la identificación, a la homogenización, a la anulación de la diferencia en todas las cosas.

Si la intensidad aparece como negada o cancelada, eso solo es porque su naturaleza es estar implicada en la extensión que la explica: "la diferencia se anula, [...] se anula en sí. Se anula en tanto que es puesta fuera de sí, en la extensión y en la cualidad que llena esa extensión. Pero a esa cualidad, como a esa extensión, las crea la diferencia. La intensidad se explica, se desarrolla, en una extensión (extensio)" (Deleuze, 2002, p. 341). Deleuze nombra como extensum todo el mundo en superficie, lleno de términos y especies, formas y partes, extensiones y cualidades, en fin, de individuos ya constituidos, de diferencias ya explicadas; y como spatium todo el mundo en profundidad, lleno de intensidades (de nivel, temperatura, presión, tensión, potencial), en fin, de campos de individuación constituyentes, de diferencias implicadas, de cantidades intensivas ${ }^{6}$.

En la misma línea, hemos de agregar que

entre la intensidad y la Idea se establece toda una corriente de intercambio, como entre dos figuras correspondientes de la diferencia. Las Ideas son

6 En termodinámica, existe una importante distinción entre propiedades métricas y no métricas que es análoga, en buena medida, a aquella entre extensión e intensidad. Las propiedades métricas pueden dividirse sin cambiar de naturaleza (por ejemplo, una misma tabla puede cortarse en medios, cuartos, u octavos y seguirá siendo la "misma" tabla). En cambio, las propiedades no métricas no pueden dividirse sin cambiar de naturaleza (por ejemplo, una olla de agua hirviendo a casi $100^{\circ} \mathrm{C}$ no puede dividirse en dos ollas de agua a $50^{\circ} \mathrm{C}$; no obstante, si esa misma agua se lleva a menos de $0^{\circ} \mathrm{C}$ se vuelve hielo y a más de $100^{\circ} \mathrm{C}$ se vuelve vapor). En ese mismo sentido, la extensión es métrica (esto es, sus diferencias son diferencias de grado) y la intensidad es no métrica (esto es, sus diferencias son diferencias de naturaleza). Véase Deleuze, 2002, pp. 355-356. 
multiplicidades virtuales, problemáticas o "perplejos", hechas de relaciones entre elementos diferenciales. Las intensidades son multiplicidades implicadas, "implejos", hechos de relaciones entre elementos asimétricos que dirigen el curso de actualización de las Ideas, y determinan los casos de solución para los problemas (Deleuze, 2002, p. 365).

La conexión entre intensidad, problema e Idea se hace evidente cuando vemos que, entre una Idea y su encarnación, entre un problema y su solución, debe haber un poder de encarnación o un poder de solución: la diferencia de intensidad. Ideas e intensidad son figuras complementarias: "[tanto] parecen anularse las cantidades intensivas como desvanercerse las Ideas problemáticas" (Deleuze, 2002, p. 365).

Para completar su ontología, Deleuze muestra la estrecha conexión entre una dialéctica de los problemas y una estética de las intensidades. En efecto, esto se logra con el aspecto de la individuación, que termina de darle sentido a la actualización de lo virtual (esto es, el paso entre diferenciación [différentiation] $\rightarrow$ individuación $\rightarrow$ dramatización $\rightarrow$ diferenciación [différenciation]):

La intensidad es lo determinante en el proceso de actualización. Es la intensidad la que dramatiza. Es ella la que se expresa inmediatamente en los dinamismos espacio-temporales de base, y la que lleva una relación diferencial, "indistinta" en la Idea, a encarnarse en una cualidad distinta y en una extensión a la que se distingue (Deleuze, 2002, pp. 366-367).

Más aún, "[el] proceso esencial de las cantidades intensivas es la individuación" (Deleuze, 2002, p. 367). Con respecto a este tema, Deleuze sigue casi literalmente los planteamientos del filósofo de la ciencia Gilbert Simondon (19241989), en especial los de su tesis de 1958: La individuación a la luz de las nociones de forma e información. Así como Deleuze expone la implicación de la intensidad y su explicación productiva en la extensión, así también Simondon denuncia hasta qué punto no se ha pensado que todos los individuos ya constituidos son producto de campos de individuación preindividuales; de modo que no tiene sentido buscar un principio de individuación en los individuos, sino que debe preguntarse por cómo lo preindividual puede engendrar lo individual. Vale la pena citar esta apropiación deleuziana de una filosofía de la individuación: 
Gilbert Simondon mostraba recientemente que la individuación supone en primer lugar un estado metaestable, es decir, la existencia de una "discordancia”, como, por lo menos, dos órgenes de magnitud o dos escalas de realidad heterogéneas entre las cuales se reparten los potenciales. Ese estado preindividual no carece de singularidades: los puntos relevantes o singulares están definidos por la existencia y la repartición de potenciales. Aparece así un campo "problemático" objetivo, determinado por la distancia entre órdenes heterogéneos. La individuación surge como el acto de solución de un problema semejante, o lo que es igual, como la actualización del potencial y la puesta en comunicación de los elementos dispares. El acto de individuación consiste, no en suprimir el problema, sino en integrar los elementos de la discordancia en un estado de acoplamiento que asegure su resonancia interna. El individuo se encuentra pues apegado a una mitad preindividual, que no es lo impersonal en él, sino más bien el reservorio de sus singularidades. Bajo todos esos aspectos, creemos que la individuación es esencialmente intensiva; y el campo preindividual, ideal-virtual, donde se forman relaciones diferenciales (Deleuze, 2002, pp. 367-368)

Entonces, la individuación simondoniana va del campo preindividual, definido por un estado metaestable donde coexisten dos órdenes diferentes de potenciales, al acto de individuación, que establece una comunicación entre los dos órdenes, definido como una resonancia interna; $a l$ individuo constituido que mantiene una "mitad" preindividual definida como una reserva de singularidades. En efecto, esto implica que los individuos nunca están perfectamente individuados; en principio, porque mantienen todavía relaciones con su "reserva de singularidades" (medio interno); en seguida, porque empiezan a interactuar con un ambiente (medio externo). Vemos también la equiparación deleuziana entre campo preindividual y condiciones de problema, asimismo entre individuos y casos de solución.

Pero falta un matiz crucial: el individuo intensivo no es lo mismo que los términos y las especies, las formas y las partes, de la extensión cualitativa. Por ejemplo, en relación con un orden orgánico,

[ese] campo intensivo de individuación determina a las relaciones que expresa a encarnarse en dinamismos espaciotemporales (dramatización), en especies que se corresponden con esas relaciones (diferenciación específica), en 
partes orgánicas que corresponden a los puntos relevantes de esas relaciones (diferenciación orgánica). Siempre es la individuación la que rige la actualización (Deleuze, 2002, p. 374).

Diríamos así que la individuación muestra el espacio y el tiempo propios de los potenciales morfogenéticos de cualquier orden. En ese sentido, el huevo es ontológicamente primario con respecto al adulto: este último jamás podría soportar las fuerzas y los movimientos que sobrelleva el primero.

Para resumir, llegamos ya al orden de las razones completo. Primero, en la diferenciación [différentiation], la Idea es una relación diferencial a la cual corresponde una cierta distribución de puntos singulares o singularidades. Segundo, en la individuación, esta relación diferencial se determina en un campo de individuación (o en unas "condiciones de problema") que presenta una disparidad entre dos órdenes de potenciales; a su vez, esta disparidad fulgura en un acto de "comunicación" que determina las singularidades (en "casos de solución”). Tercero y cuarto, en la dramatización y la diferenciación [différenciation], estas diferencias de intensidad (disparidades) "dramatizan" la especificación (esto es, la diferenciación específica) de las especies y la partición (esto es, la diferenciación orgánica) de las partes. Al final, vemos que la ontología deleuziana expuesta en Diferencia y repetición (2002) es de cabo a rabo "problemática": las Ideas problemáticas son verdaderas estructuras genéticas, en tanto su determinación progresiva y divergente, sus "soluciones", constituye la producción de las cosas mismas.

\section{Consideraciones finales}

LAS LÍNEAS DE PENSAMIENTO abordadas por este artículo obligan a tomar en cuenta una particularidad de la escritura de Diferencia y repetición (2002). Este texto se constituye, por una parte, como una obra ecléctica de gran aliento, y, por otra parte, como un tratado medieval sistemático. Esta doble fuerza es difícil de mantener en su comprensión: se evidencia en la manera como las lecturas exegéticas posteriores tienden a exaltar un aspecto del libro como "el" aspecto crucial, en vez de lidiar con la complejidad de seguir cuidadosamente una hebra por todos sus nudos. Este artículo, por el contrario, se esfuerza por mantener, a modo de haltera, los dos pesos al tiempo: los problemas son un tema más del libro a la vez que un eje transversal a toda la filosofía de Deleuze. 
En tanto tema, los problemas por sí solos son suficientemente interesantes. En primer lugar, la intención explícita de remover el lastre subjetivo y negativo con el que cargan los problemas se vuelve una postura filosófica importante. $\mathrm{Ni}$ vacíos por llenar, ni obstáculos por superar, ni dudas por resolver: los problemas no se identifican con una insuficiencia epistemológica temporal; para Deleuze, en cambio, debe relucir su valor objetivo y positivo o, mejor, ontológico. En principio, esta revaluación no depende tanto de la historia de la filosofía como de otras fuentes esotéricas (la metafísica del cálculo diferencial) y científicas (la teoría matemática de los problemas, la geometría no euclidiana, la termodinámica). No obstante, Deleuze, como de costumbre, tiene una lectura completamente idiosincrática de los autores: las ilusiones de la razón en Kant muestran la importancia de una facultad de plantear problemas; asimismo, Kant establece la complementariedad entre Ideas y problemas; con Bordas-Demoulin reluce el carácter universal de las Ideas-problemas: ni generales, ni particulares, ni objetos de la experiencia, ni conceptos del entendimiento; con Maïmon y Hoëne-Wronski aparece el potencial genético de las Ideas-problemas, o la forma como sus relaciones diferenciales y sus puntos singulares (aunque ideales) son tan reales como las especies y las partes producidas por su actualización; con Carnot, Abel y Galois -y, sobre todo, con su síntesis en Lautman- la noción en principio matemática de problema (como problema, condiciones de problema y campo de soluciones) adquiere un vestido dialéctico como estructura del pensar mismo: las Ideasproblemas son a la vez trascendentes con respecto a sus encarnaciones-soluciones (por lo tanto no desaparecen en su encarnación-solución) e inmanentes (por lo tanto continúan determinándose progresivamente en series de encarnacionessoluciones); finalmente, con Riemann, las Ideas-problemas se describen como multiplicidades o variedades: objetos ideales, multidimensionales, y -es lo más importante- no definidos ya por su inscripción en un plano superior (trascendente) sino definidos por sus relaciones de vecindad (inmanentes). Hasta aquí, hay una profunda y heterodoxa teoría de los problemas que, aunque bebe de muchas fuentes, es propiamente deleuziana.

En tanto eje transversal, las Ideas-problemas van más allá de una simple temática en Diferencia y repetición (2002). De hecho, se vuelven el elemento fundacional de toda la ontología deleuziana plausiblemente nombrada como una teoría de la actualización de las Ideas. Deleuze postula como operación ontológica 
por excelencia el planteamiento y la solución progresiva de problemas, o, lo que es igual, la encarnación de las Ideas en especies y partes divergentes. La arquitectura conceptual es totalmente isomórfica en este punto:

\begin{tabular}{|l|l|}
\hline Problema & $\begin{array}{l}\text { Idea, o relaciones diferenciales y puntos singulares } \\
\text { diferenciados [différentiées] }\end{array}$ \\
\hline Condiciones de problema & $\begin{array}{l}\text { Campos de intensidades preindividuales y sujetos } \\
\text { larvales individuados }\end{array}$ \\
\hline Campos de solución & Dinamismos espaciotemporales dramatizados \\
\hline Soluciones & Especies y partes diferenciadas [différenciées] \\
\hline
\end{tabular}

Al final, pues, el artículo esclarece este paralelismo problema-Idea sin el cual no puede haber una comprensión detallada de la ontología deleuziana en Diferencia y repetición. Primero, plantear un problema es tener una Idea: un plano de relaciones diferenciales y puntos singulares. Segundo, dar las condiciones de problema es mostrar en qué órdenes-problemáticos (o campos de intensidades preindividuales) y en qué dominios-problemáticos (o sujeto larvario) se puede definir el problema (o dar el primer paso en la actualización de la Idea). Tercero, abrir campos de solución es hacer fulgurar los espacios y los tiempos propios de la actualización (antes de que haya especies hay una especiación, antes de que haya partes hay una partición). Cuarto, ofrecer soluciones a un problema es exponer a las especies y a las partes como las divergentes encarnaciones de la Idea. Por tanto, toda ontogénesis es la solución a un problema, es decir, la actualización de una Idea.

\section{Referencias}

Bahoh, J. (2019). Deleuze's Theory of Dialectical Ideas: The Influence of Lautman and Heidegger. Deleuze and Guattari Studies, 13(1), 19-53. DoI: https://doi.org/10.3366/dlgs.2019.0340

Bianco, G. (2018). The Misadventures of the "Problem" in "Philosophy", from Kant to Deleuze. Angelaki, 23(2), 8-30. Dor: https://doi.org/10.1080/096 9725X.2018.1451459 
Deleuze, G. (2002). Diferencia y repetición. (Trads. M.S. Delpy \& H. Beccacece). Buenos Aires: Amorrortu.

Deleuze, G. (2017). El bergsonismo. (Trad. P. Ires). Buenos Aires: Cactus.

Deleuze, G., \& Parnet, C. (1980). Diálogos. (Trad. J. Vázquez). Valencia: PreTextos.

Duffy, S. B. (2009). Deleuze and the Mathematical Philosophy of Albert Lautman. En: J. Roffe \& G. Jones (Eds.), Deleuze's Philosophical Lineage (pp. 356-379). Edinburgo: Edinburgh University Press.

During, E. (2004). 'A History of Problems': Bergson and the French Epistemological Tradition. Journal of the British Society for Phenomenology, 35(1), 4-23. DoI: https://doi.org/10.1080/00071773.2004.11007419

González Geraldino, F. E. (2018). Las funciones como problemas: un encuentro entre Luhmann-Deleuze. Revista del Magister en Análisis Sistémico Aplicado a la Sociedad, 38, 22-37. DoI: 10.5354/0719-0527.2018.51036

Wasser, A. (2017). How Do We Recognise Problems? Deleuze and Guattari Studies, 11(1), 48-67. Dor: https://doi.org/10.3366/dls.2017.0251 